\title{
Villoglandular Papillary Adenocarcinoma With Cervical Intraepithelial Neoplasia 3 of the Cervix in a Woman With a Misdiagnosis of a Cervical Polyp
}

\author{
Remi Hasegawa-Nakajima ${ }^{\text {a, }}$, Hisami Kiseki ${ }^{\text {a }}$, Shun-ichi Ikeda a, b, c
}

\begin{abstract}
We report a case in which a polypoid lesion with a diameter of $5 \mathrm{~mm}$ was diagnosed as a cervical polyp due to a negative Papanicolaou (Pap) smear in the cervix, and polypectomy revealed a diagnosis of villoglandular papillary adenocarcinoma (VGA) on histopathological examination. A 63-year-old woman with a cervical polyp, who had no abnormal symptoms and a negative Pap smear was referred to our hospital. We performed cervical polypectomy, and the pathological result was VGA with cervical intraepithelial neoplasia (CIN) 3. Even if an asymptomatic cervical polyp with a negative Pap smear is diagnosed, in some patients VGA of the uterine cervix may coexist with CIN. Therefore, asymptomatic cervical polyps with negative Pap smears should not be followed up without removal.
\end{abstract}

Keywords: Uterine cervical polyp; Villoglandular papillary adenocarcinoma; Cervical intraepithelial neoplasia

\section{Introduction}

Villoglandular papillary adenocarcinoma (VGA) is a rare malignant tumor of well-differentiated cervical adenocarcinoma showing abnormal vaginal bleeding or an abnormal Papanicolaou (Pap) smear [1]. It macroscopically shows papillary or polypoid lesions. We encountered a case diagnosed as a cervical polyp because of a negative Pap smear without abnormal vaginal bleeding.

Cervical polypectomy and histopathological examination revealed that VGA coexisted with cervical intraepithelial neoplasia (CIN) 3. In our patient, histopathological examination of

Manuscript submitted September 22, 2020, accepted October 6, 2020

Published online October 21, 2020

aDepartment of Obstetrics and Gynecology, Kohseichuo General Hospital, Tokyo, Japan

${ }^{b}$ Both authors contributed equally to this manuscript.

${ }^{\mathrm{c} C}$ Corresponding Author: Shun-ichi Ikeda, Department of Obstetrics and Gynecology, Kohseichuo General Hospital, 1-11-7, Mita, Meguro-ku, 153-8581,

Tokyo, Japan. Email: shiked@kohseichuo.jp

doi: https://doi.org/10.14740/jmc3589 a clinically diagnosed cervical polyp revealed VGA with CIN3

\section{Case Report}

A 63-year-old woman, who had no abnormal signs or symptoms such as genital bleeding, underwent a cervical cancer screening examination at her family clinic. She had no history of using oral contraceptives. A pelvic examination revealed a cervical polyp with a diameter of $5 \mathrm{~mm}$, which had a negative Pap smear. She was referred to our hospital for follow-up of a cervical polyp. In our hospital, cervical polypectomy was performed after the Pap smear.

The results of the Pap smear were also negative, but the pathological result of the polypectomy was VGA with CIN3 (Fig. 1a). VGA's papillae were thin and short (Fig. 1b), and the CIN3 lesion existed with adenocarcinoma (Fig. 2a). The CIN3 lesion showed that neoplastic cells replaced the upper third of the squamous epithelium (Fig. 2b).

Next, we performed a cone biopsy. No papillary lesions of VGA were observed in the resected cervix on cone biopsy, and adenocarcinoma in situ (AIS) that appeared to correspond to the basal part of VGA was observed (Fig. 3). She worried about the recurrence of cervical adenocarcinoma and requested a hysterectomy. We performed a total abdominal hysterectomy with bilateral salpingo-oophorectomy and confirmed that there were no residual tumors in the uterus.

\section{Discussion}

VGA occurs most often in women younger than 40 years of age. Since it has macroscopic lesions, it shows abnormal genital bleeding and abnormal cytology [1]. A cone biopsy is required to diagnose VGA. Since VGA has an infiltrating shallow lesion, it is possible to preserve fertility by performing a cone biopsy $[2,3]$. Moreover, VGA has been pointed out to be related to a history of taking oral contraceptives [2].

We describe our experience with a 63-year-old woman who had VGA without a history of using oral contraceptives. Our case showed the following three characteristics. First, VGA may show negative cytology. Second, VGA may coexist with CIN. Third, asymptomatic VGA may show the same macroscopic findings as an asymptomatic cervical polyp. 




Figure 1. VGA obtained by polypectomy. (a) Whole mounted view of cervical exophytic tumor (hematoxylin and eosin (H\&E) stain, $\times 1$ ). (b) The papillae are short, covered by atypical endocervical epithelia, in a villoglandular lesion (H\&E stain, $\times 4)$. VGA: villoglandular papillary adenocarcinoma.

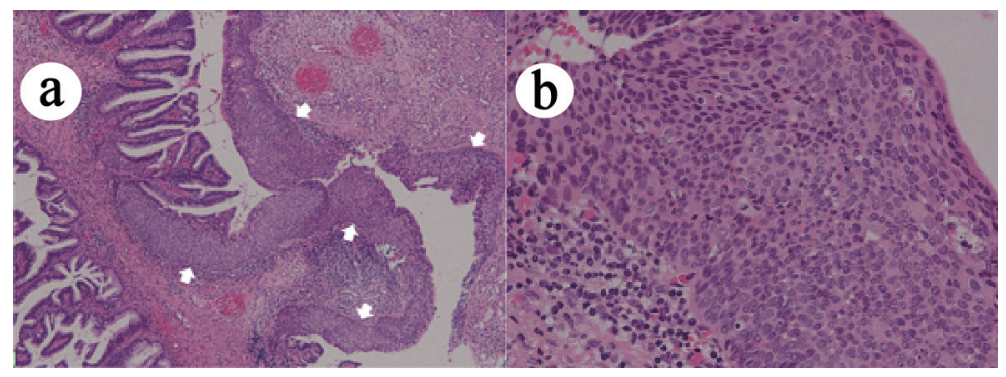

Figure 2. CIN3, coexisted with adenocarcinoma. (a) CIN3 lesion (indicated by white arrows) existed with adenocarcinoma (hematoxylin and eosin (H\&E) stain, $\times 4)$. (b) CIN3 lesion shows severe atypia reaching the upper third of the squamous epithelium $(H \& E$ stain, $\times 20)$. CIN: cervical intraepithelial neoplasia.

Our patient with VGA was clinically considered a cervical polyp with a negative Pap smear. Many cervical polyps are asymptomatic and are usually found by chance on pelvic examination. Galon et al analyzed 362 women with macroscopically diagnosed cervical polyps in the 1900 s, and divided them into symptomatic and asymptomatic groups [4]. No malignant tumors were detected in the asymptomatic group. MacKenzie et al also reviewed the pathological reports of 1,366 specimens

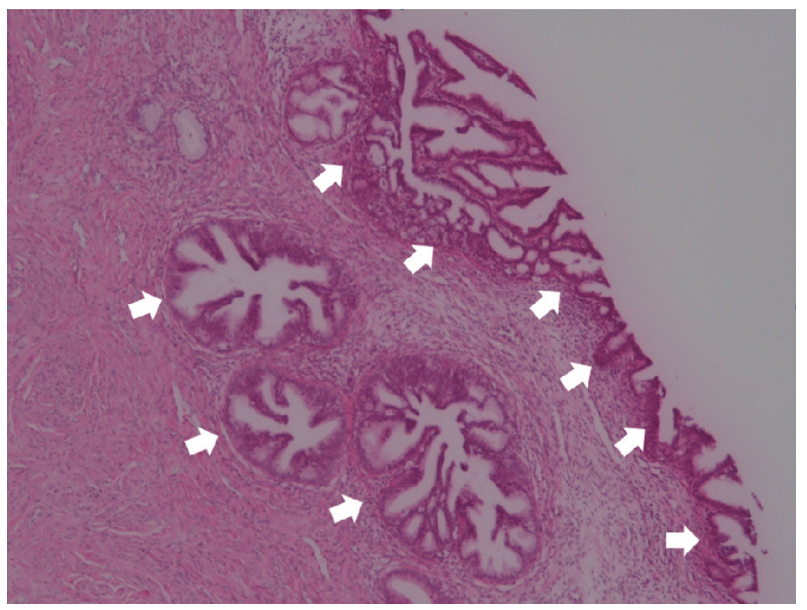

Figure 3. Residual AIS lesion. Residual AIS lesion (indicated by white arrows) that appeared to correspond to the basal part of VGA was observed after cone biopsy (hematoxylin and eosin (H\&E) stain, $\times 4$ ). AIS: adenocarcinoma in situ; VGA: villoglandular papillary adenocarcinoma. resected as cervical polyps [5]. Four hundred five cases (33\%) with genital bleeding or abnormal cytology were considered the symptomatic group, and 919 cases $(67 \%)$ were designated as the asymptomatic group. The two groups were analyzed together with the cost of the test. No malignant tumor was found in any of the asymptomatic group.

These results show that there is no reason to aggressively remove asymptomatic polyps, and if not removed, asymptomatic cervical polyps would result in significant medical cost savings. Nelson et al concluded in their paper that regular removal of cervical polyps in asymptomatic women with normal cervical cytology had little impact on management and might be detrimental [6].

However, the accuracy rate of adenocarcinoma is known to be significantly lower than that of squamous cell carcinoma. Srisomboon et al examined the preoperative cytological characteristics of 75 patients with AIS diagnosed by conization. They showed that glandular abnormalities were found in only $37.3 \%$ of the patients [7]. Krane et al also showed that the sensitivity of cytology for glandular diseases was $45 \%$ [8]. Furthermore, squamous cell abnormalities can be detected with a high frequency by cytology. Krane et al reported that adenocarcinoma cells potentially mistaken for intimal cells in the lower uterus or cervical cells with tubo-endometrioid metaplasia were reasons for the lower detection rate of glandular cell abnormalities as compared with squamous epithelium abnormalities. Another reason is that squamous epithelial cells have lesions on the epithelial surface, whereas glandular epithelial cells may have lesions in crypts [9]. Therefore, atypical 
glandular cells in Pap smears may be diagnosed as adenocarcinoma. Moreover, it is said that VGA is a well-differentiated cancer, which may lead to minimal atypia on cytodiagnosis and not lead to the diagnosis of VGA [10].

In our patient, Pap smear was performed twice by her family clinic and our hospital, and the cytological results were all diagnosed as negative intraepithelial lesions for malignancy (NILM). It was speculated that the reason why CIN was not detected by Pap smear was that CIN existed between the villoglandular lesions. When a lesion considered to be a cervical polyp is found macroscopically, cervical polypectomy should be performed even if there is no abnormality in the Pap smear. It is necessary to rule out malignancy by pathological examination.

Multiple biopsies of polypoid lesions are required because VGA can coexist with CIN. Our patient had CIN3 in VGA. Depending on the biopsy site, the preoperative diagnosis may be mistaken if there is a concomitant lesion. Korach reported nine patients in whom VGA was confirmed in final pathology findings [11]. Only two of these nine patients had a correct preoperative diagnosis. The preoperative diagnosis in three of the nine patients was cervical polyps, cervical dysplasia, and CIN3, but not VGA. The preoperative diagnosis in the remaining four patients was cervical adenocarcinoma in three patients and endometrial cancer in one patient. Zhou et al reported three cases of VGA, and one case had a preoperative diagnosis of CIN1 [12]. Among the cases reported by Korach and Zhou et al, there were cases in which CIN was initially diagnosed. This was considered a misdiagnosis because CIN was present in VGA, and only CIN was biopsied. If cancer is diagnosed as benign such as CIN, the start of treatment is delayed. Multiple biopsies are needed for large lesions that cannot undergo polypectomy to prevent a delay in diagnosis.

Asymptomatic VGA may show macroscopic findings similar to asymptomatic cervical polyps. In our patient, the lesion size was as small as $5 \mathrm{~mm}$, and the microscopic structure of the villoglandular lesion was short and not long. These two findings may not have led to the vaginal bleeding symptoms. As in this case, VGA of a polypoid lesion clinically diagnosed as a cervical polyp may be asymptomatic despite being malignant. When an asymptomatic cervical polyp is encountered, examination should proceed, bearing the possibility of malignancy in mind.

In summary, we described our experience with VGA, which was clinically considered a cervical polyp with a negative Pap smear. Even if a cervical polyp-like lesion has no clinical symptoms with a negative Pap smear, immediately designating it as a cervical polyp has the risk of missing cancer. VGA has few malignant findings on a Pap smear. Since there was a VGA showing a polyp lesion like this case, it is necessary to confirm the pathological diagnosis by performing a biopsy even with negative PAP smears. VGA may also be associated with CIN, so if all lesions cannot be resected by polypectomy, one biopsy may collect only CIN lesions, even with VGA. Therefore, multiple biopsies are often required.

\section{Acknowledgments}

None to declare.

\section{Financial Disclosure}

None to declare.

\section{Conflict of Interest}

None to declare.

\section{Informed Consent}

Informed consent was obtained from the patient prior to completing the case report.

\section{Author Contributions}

RHN and SiI contributed to the writing of the manuscript. RHN, HK and SiI reviewed and adjusted the manuscript prior to submission.

\section{Data Availability}

The data supporting the findings of this study are available from the corresponding author upon reasonable request.

\section{References}

1. Mutter GL, Prat J. Pathology of the female reproductive tract. 3rd ed. Churchill Livingstone: Elsevier; 2014, p. 265-267.

2. Jones MW, Silverberg SG, Kurman RJ. Well-differentiated villoglandular adenocarcinoma of the uterine cervix: a clinicopathological study of 24 cases. Int J Gynecol Pathol. 1993;12(1):1-7.

3. Jones MW, Kounelis S, Papadaki H, Bakker A, Swalsky PA, Woods J, Finkelstein SD. Well-differentiated villoglandular adenocarcinoma of the uterine cervix: oncogene/tumor suppressor gene alterations and human papillomavirus genotyping. Int J Gynecol Pathol. 2000;19(2):110-117.

4. Golan A, Ber A, Wolman I, David MP. Cervical polyp: evaluation of current treatment. Gynecol Obstet Invest. 1994;37(1):56-58.

5. MacKenzie IZ, Naish C, Rees CM, Manek S. Why remove all cervical polyps and examine them histologically? BJOG. 2009;116(8):1127-1129.

6. Nelson AL, Papa RR, Ritchie JJ. Asymptomatic cervical polyps: can we just let them be? Womens Health (Lond). 2015;11(2):121-126.

7. Srisomboon S, Tantipalakorn C, Charoenkwan K, Srisomboon J. Cervical Screening Results Leading to Detection of Adenocarcinoma in Situ of the Uterine Cervix. Asian Pac J Cancer Prev. 2019;20(2):377-382.

8. Krane JF, Granter SR, Trask CE, Hogan CL, Lee KR. Papanicolaou smear sensitivity for the detection of ad- 
enocarcinoma of the cervix: a study of 49 cases. Cancer. 2001;93(1):8-15.

9. Dalbert DB, Rodriguez de la Pena MM, Falabella P, Figueredo A, Borelli A, Murai J, et al. Hidden carcinoma of uterine cervix. Clin Obstet Gynecol Reprod Med. 2016;2:128-132.

10. Chang WC, Matisic JP, Zhou C, Thomson T, Clement PB, Hayes MM. Cytologic features of villoglandular adenocarcinoma of the uterine cervix: comparison with typical endocervical adenocarcinoma with a villoglandu- lar component and papillary serous carcinoma. Cancer. 1999;87(1):5-11.

11. Korach J, Machtinger R, Perri T, Vicus D, Segal J, Fridman E, Ben-Baruch G. Villoglandular papillary adenocarcinoma of the uterine cervix: a diagnostic challenge. Acta Obstet Gynecol Scand. 2009;88(3):355-358.

12. Zhou QY, Chen HY, Yang SM, Li YH, Wu XQ. Villoglandular papillary adenocarcinoma of the uterine cervix: A report of 4 cases and a review of the literature. Oncol Lett. 2016;11(1):837-841. 